\title{
Functional Electrical Stimulation with Neural Network Controlled State Feedback
}

\author{
J. Beckmann, W.J. Daunicht, V. Hömberg
}

Neurologisches Therapiecentrum at the Heinrich-Heine-University Düsseldorf, FRG

Optimal linear control: As described earlier, we proposed the use of artificial neural networks for the control of free stance and simple movements in spinal cord injured people by Functional Electrical Stimulation (FES). For simulation purposes we presently use a planar patient model with 3 segments (angles $\theta$ ) and 6 muscles [1].

With the state vector $\boldsymbol{x}$ and the command vector $\boldsymbol{u}$ of stimulation currents the nonlinear dynamics of this model can be denoted as: $\dot{\boldsymbol{x}}=f(\boldsymbol{x}, \boldsymbol{u})$. In the theory of optimal linear control a state feedback approach, $u:=\boldsymbol{K} \cdot \boldsymbol{x}+\boldsymbol{V} \cdot \boldsymbol{\theta}_{\text {desired }}$, is proposed for the linearized dynamics. $K$ is determined in order to minimize the following performance index with positive definite cost-matrices $\boldsymbol{Q}, \boldsymbol{R}[2]$ :

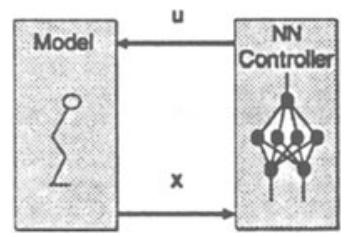

$$
E:=\int_{0}^{\infty} d t\left(e^{\alpha t} \cdot \boldsymbol{x}^{T} \cdot Q \cdot \boldsymbol{x}+\boldsymbol{u}^{T} \cdot \boldsymbol{R} \cdot \boldsymbol{u}\right)
$$

Results: A reference path $\theta_{R}(\tau)$ forming a continuous movement of standing up from a chair followed by kneeling down was chosen such that itself and its surroundings can be considered as a purposeful working range (cf. left figure below). Next, the state feedback matrices $\boldsymbol{K}\left(\boldsymbol{\theta}_{R}\right), \boldsymbol{V}\left(\boldsymbol{\theta}_{R}\right)$ were determined at different locations $\boldsymbol{\theta}_{R}(\tau)$ of the reference path. The resulting data-set was then implemented into a neural network. Our network uses radial basis functions and a normalization procedure at the output [1]. In a simple controller test the points of the reference path were presented in a time sequence yielding a desired trajectory that should be tracked. No single state feedback controller with constant $K, V$ - matrices was able to track the trajectory. In contrast, the neural network controller that comprises the knowledge of gain matrices along the whole reference path correctly tracked the path. A similar success of the network could be observed for other tasks.
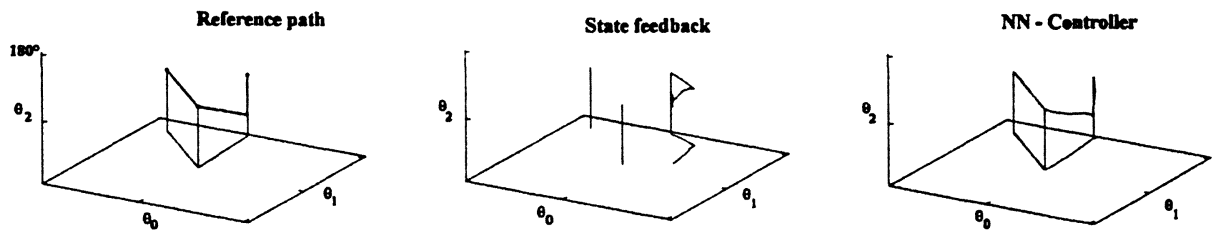

\section{References}

1. J. Beckmann, W.J. Daunicht and V. Hömberg, Control of a Paraplegic Patient Model by Neuroprothetic Networks, in: I. Aleksander, J.Taylor (eds.): Artificial Neural Networks II, pp.471-474 (1992).

2. T. Kailath, Linear Systems, Prentice-Hall (19S0).

Acknowledgements: This work was supported by DFG grant Da 199/2-1. 